\title{
Influência do treinamento físico sobre parâmetros do eixo hipotála- mo-pituitária-adrenal de ratos administrados com dexametasona
}

\author{
J. Rodrigo Pauli \\ José Leme \\ Daniel Crespilho \\ M. Alice Mello \\ Gustavo Rogatto \\ Eliete Luciano
}

\author{
Universidade Estadual Paulista \\ Campus de Rio Claro \\ Instituto de Biociências \\ Departamento de Educação Física \\ Brasil
}

\section{RESUMO}

O objetivo do presente estudo foi verificar os efeitos do exercício físico agudo e crônico sobre o eixo hipotálamo-pituitáriaadrenal de ratos administrados com dexametasona. Ratos Wistar jovens foram divididos em quatro grupos: controle sedentário (CS), controle treinado (CT), dexametasona sedentário (DxS) e dexametasona treinado (DxT). O protocolo de treinamento consistiu de natação 1 hora/dia, 5 dias/semana, durante 10 semanas, suportando uma sobrecarga relativa a $5 \%$ do seu peso corporal. A dexametasona foi administrada 5 dias/ semana $(2 \mu \mathrm{g} /$ dia diluída em $150 \mu \mathrm{l}$ de $\mathrm{NaCl}-0,9 \%)$. Antes do sacrifício os ratos receberam insulina subcutânea para o cálculo da remoção máxima de glicose. No final do período experimental amostras de sangue foram obtidas para determinação da glicose e hormônio adrenocorticotrófico (ACTH) séricos. Amostras do músculo gastrocnêmio, da glândula adrenal e tecido adiposo epididimal foram utilizadas para determinação do peso, teor de glicogênio e ácido ascórbico, respectivamente. Nossos resultados indicam que a exposição crônica a dexametasona está associada com diminuição da sensitividade à insulina. O tratamento com dexametasona diminuiu a secreção de ACTH em resposta ao exercício agudo, mostrando diferença no funcionamento do eixo CRH-ACTH-adrenal entre os grupos estudados. Em conclusão, o exercício pode preponderar sobre o feedback negativo da dexametasona na ativação do eixo hipotálamo-pituitária-adrenal.

Palavras-chave: treinamento físico, dexametasona, resistência à insulina, eixo hipotálamo-pituitária-adrenal.

\begin{abstract}
Influence of physical training on hypothalamo-pituitary-adrenal axis parameters of rates administered with dexametasone

The aim of present study was to investigate the influence of acute and chronic physical exercise on the hypothalamo-pituitary-adrenal axis in Wistar rats administered with dexamethasone. Young Wistar rats were divided into four groups: sedentary control (CS), sedentary dexametha-sone (DxS), trained control (CT) and trained dexamethasone (DxT). Training protocol consisted of swimming $1 \mathrm{~h} /$ day, 5 days/week, during 10 weeks, supporting a load of 5\% of their body weight. Dexamethasone was administered 5 times for week $(2 \mu \mathrm{g} /$ day in $150 \mu \mathrm{l} 0,9 \% \mathrm{NaCl}$ ). Before sacrifying the rats each received a subcutaneous insulin to calculate the maximum decreased in blood glucose. Venous blood samples were obtained at the end of the experimental period to determine serum glucose and ACTH.

Gastrocnemius, adrenal and adi-pose epididimal tissues' samples were used to determine weight, glyco-gen and ascorbic acid concentration, respectively. Data suggests that chronic exposure to dexamethasone was associated with decreased insulin sensitivity. The dexametasona treatment decreased ACTH release in response to acute exercise, showing marked differences in the functioning of the $\mathrm{CRH}$ - $\mathrm{ACTH}$ adrenal axis between groups of rats. In conclusion, exercise can override the dexamethasone negative feedback of hypothalamopituitary-adrenal axis activation in rats.
\end{abstract}

Key Words: physical training, dexamethasone, insulin resistance, hypothalamo-pituitary-adrenal axis. 


\section{INTRODUÇÃo}

A relevância do estudo da resistência periférica à insulina é indiscutível, visto que está associada a diversas patologias como diabetes mellitus não-insulino-dependente, obesidade e hipertensão (1). A resistência ao hormônio pode ser decorrente de níveis elevados de glicocorticóides, hormônio do crescimento e catecolaminas. A resistência dos tecidos à insulina tem sido estudada em modelos experimentais por meio da administração de glicocorticóides, como a dexametasona, o que facilita a investigação dos mecanismos fisiológicos envolvidos nos referidos processos $(2,3)$.

A resistência periférica ao hormônio acaba afetando o metabolismo de carboidratos, lipídeos e proteínas, resultando em hiperglicemia, dislipidemias, atrofia muscular, entre outros distúrbios metabólicos. Estas alterações sobre o metabolismo podem provocar diversas complicações, tais como problemas cardiovasculares, aterosclerose, elevação da pressão arterial e disfunções renais (4).

As desordens metabólicas que ocorrem em organismos insulino resistentes podem exercer efeitos também sobre estruturas envolvidas no mecanismo de respostas ao estresse, como o eixo hipotálamo-pituitária-adrenal (HPA). Por outro lado, o treinamento físico exerce efeitos positivos sobre o organismo, podendo promover alterações endócrino-metabólicas mesmo nas condições adversas, como na resistência à insulina, na ausência de hormônios da hipófise anterior, entre outras, auxiliando na homeostase corporal. Além dos efeitos sobre o metabolismo intermediário, o treinamento físico exerce influência sobre o eixo HPA. Tal eixo é ativado pelo estresse e inibido pelos glicocorticóides. Vários tipos de estresse estimulam a liberação do ACTH, modificando sua ritmicidade de secreção normal. Estresse físico, emocional e químico como dor, trauma, hipóxia, hipoglicemia aguda, exposição ao frio, cirurgia e depressão, estimulam a secreção de ACTH e cortisol. O uso de altas doses de glicocorticóides sintéticos pode suprimir a secreção de ACTH e a retirada abrupta pode encontrar o organismo deficiente em cortisol endógeno (5). Neste sentido, o treinamento físico pode exercer efeitos positivos sobre o eixo HPA de organismos submetidos à administração crônica de glicocorticóides, já que nessa situação de estresse pode permanecer a liberação de ACTH (6,
7). Deuster et al. (8) observaram que homens saudáveis exibiam significativo aumento na concentração de ACTH e cortisol em resposta ao exercício de alta intensidade após tratamento com dexametasona. Entretanto, os possíveis mecanismos pelos quais o exercício físico prepondera sobre o feedback negativo do glicocorticóide e aumenta a responsividade do eixo HPA não são totalmente conhecidos. Diante dos estudos encontrados na literatura, verifica-se a necessidade de realização de trabalhos que esclareçam os mecanismos envolvidos no papel do exercício físico sobre o eixo hipotálamo-pituitáriaadrenal, bem como desordens metabólicas decorrentes do uso de glicocorticóides. Assim, o objetivo deste estudo foi verificar os efeitos do exercício físico agudo e crônico sobre parâmetros funcionais do eixo hipotálamo-pituitária-adrenal de ratos administrados com dexametasona.

\section{MATERIAIS E MÉTODO}

\section{Animais e seu tratamento}

Para o desenvolvimento deste trabalho utilizamos ratos machos jovens Wistar (Rattus Norvegicus Albinus Wistar) com aproximadamente 65 dias. Os animais, provenientes do Biotério Central da UNESP, Botucatu, foram mantidos no Biotério do Laboratório de Biodinâmica do Departamento de Educação Física, do Instituto de Biociências da UNESP, Rio Claro. Os animais foram alimentados com ração balanceada padrão (Purina) e água "ad libitum" e distribuídos em gaiolas coletivas (com 4 ratos por gaiola) à temperatura ambiente controlada de $25^{\circ} \mathrm{C}$ e fotoperíodo de $12 \mathrm{~h}$ claro/ $12 \mathrm{~h}$ escuro. Todos os experimentos com os animais foram realizados de acordo com a "European Convention for Protection of Vertebrate Animals used for Experimental and other Scientific Purposes" (Council of Europe $n^{\circ} 123$, Strasburg, 1985).

\section{Delineamento e grupos experimentais}

Os animais foram distribuídos aleatoriamente em quatro grupos de 10 ratos denominados:

Controle Sedentário (CS): constituído de ratos normais que não foram submetidos ao protocolo de treinamento físico e/ou administração de dexametasona; Controle Treinado (CT): constituído de ratos normais que foram submetidos somente ao protocolo de treinamento físico; 
Dexametasona Sedentário (DxS): constituído de ratos que foram submetidos somente à administração de dexametasona; e

Dexametasona Treinado (DxT): ratos que foram submetidos ao protocolo de treinamento físico e à administração de dexametasona.

\section{Protocolo de treinamento}

O protocolo de exercício consistiu de natação 5 vezes/semana, 1 hora/dia, com sobrecarga de $5 \%$ em relação ao peso corporal, que foi acoplada com elástico ao tronco dos animais. O período de treinamento perdurou por 10 semanas, coincidentes com a administração da dexametasona.

Período de adaptação: $\mathrm{Na} 1^{\text {a }}$ semana os ratos iniciaram o treinamento nadando 20 minutos sem nenhuma sobrecarga adicional ao seu peso corporal, sendo, então, acrescentados $10 \mathrm{~min} /$ dia de exercício de natação, até completar 1 hora por sessão. Após esse período de adaptação, foi acrescentada a sobrecarga ao treinamento, sendo esta semanalmente aferida e ajustada em 5\% em relação ao peso do animal.

Local de treinamento: As sessões de natação foram realizadas em tanque de amianto com $100 \mathrm{~cm}$ de comprimento, $70 \mathrm{~cm}$ de largura e $60 \mathrm{~cm}$ de altura, contendo água numa profundidade de $40 \mathrm{~cm}$, para evitar que os ratos apoiassem a cauda no fundo do recipiente.

Foram colocados no máximo 8 animais nadando ao mesmo tempo em cada recipiente. A temperatura da água foi mantida entre $31^{\circ}$ e $32^{\circ} \mathrm{C}$ por ser considerada termicamente neutra em relação à temperatura corporal do rato (9). As sessões de treino eram realizadas sempre no final da tarde entre 17:00 e 18:00 horas.

\section{Administração de dexametasona}

A dexametasona (DEXA) foi administrada na concentração de $2 \mu$ g diluído em $150 \mu \mathrm{l}$ de $\mathrm{NaCl}-0,9 \%$, via subcutânea, 5 dias por semana, durante 10 semanas consecutivas. A droga foi injetada diariamente, às 8:00 horas. O uso de baixas doses de dexametasona na ordem de microgramas por dia é sugerida por Severino et al. (1).

\section{Avaliações}

Avaliações prévias ao sacrifício dos animais na condição de repouso

Durante as 10 semanas de estudo foi avaliado sema- nalmente o peso corporal dos animais, registrado em planilha eletrônica. Para averiguação da evolução do peso foi realizada a subtração entre o peso final e inicial obtido em cada grupo experimental.

Para a caracterização da resistência à insulina foi realizado na $9^{a}$ semana de experimento o teste de tolerância à insulina (KITT) após 36 horas da última sessão de exercício e sob abstinência da dexametasona no mesmo intervalo de tempo. Os ratos receberam insulina mista regular purificada via subcutânea (30mU/100g de peso corporal). As amostras de sangue foram coletadas nos tempos 0,30 e 60 minutos e utilizadas para a dosagem de glicose (método da glicose oxidase). Com os valores obtidos nos tempos 0 e 60 foi calculada a taxa de desaparecimento da glicose sanguínea. A glicose sérica (t $1 / 2)$ foi calculada por meio dos mínimos quadrados das concentrações de glicose após a administração de insulina (10). Avaliações após o sacrifício dos animais na condição de repouso

Ao final do período experimental, os animais foram mantidos em repouso por 36 horas em relação à última sessão de exercício, sem jejum prévio. No mesmo período não foi administrada a dexametasona. Após o sacrifício dos animais foram retiradas amostras teciduais e de sangue para serem avaliadas. Análises sanguíneas

Foi determinada a glicose sérica através do método enzimático colorimétrico da glicose oxidase-peroxidase (11). O hormônio adrenocorticotrófico (ACTH) foi determinado através do método de radioimunoensaio de fase sólida, Kit Coat-A-Count da Diagnostic Products Corporation (DPC-USA). Análises teciduais

O glicogênio muscular foi avaliado pelo método fenol em meio ácido descrito por Dubois et al. (12), com posterior leitura em espectrofotômetro. O tecido adiposo epididimal foi pesado utilizando-se balança analítica. O peso relativo foi determinado dividindo-se o valor obtido na pesagem pelo peso total do animal no dia do sacrifício.

Depois de retirada e dissecada sobre um papel de filtro umedecido em solução salina, foi feita a pesagem em balança analítica, das glândulas adrenais. O peso relativo foi determinado dividindo-se o valor obtido na pesagem pelo peso total do animal no dia do sacrifício. A glândula adrenal esquerda foi retirada e mace- 
rada para a determinação do conteúdo de ácido ascórbico, já que o consumo deste ácido está relacionado com a utilização na síntese dos hormônios do estresse. A concentração de ácido ascórbico foi medida a 520nm contra curva padrão de ácido ascórbico (13). Avaliações após a sessão aguda de exercício físico

A razão deste segundo estudo foi avaliar a funcionalidade do eixo hipotálamo-pituitária-adrenais, nesse modelo experimental, imediatamente após a realização de uma sessão aguda de exercício físico. Para isso, todo procedimento metodológico utilizado foi idêntico aos citados anteriormente. Quanto ao sacrifício dos animais, ao contrário do experimento anterior que foi realizado após um repouso de 36 horas, nessa etapa os ratos foram sacrificados após a realização de uma sessão aguda de exercício de natação com duração de 1 hora, que foi realizada sem qualquer sobrecarga adicional ao seu peso corporal. Imediatamente ao término do esforço ocorreu o sacrifício dos animais, para a realização das seguintes análises laboratoriais: ACTH, ácido ascórbico e peso fresco da adrenal, conforme descrito anteriormente.

\section{Análise estatística}

A análise estatística foi feita por ANOVA e aplicação do teste de post-hoc de Newman-Keuls, onde adequado, com nível de significância pré-fixado em $5 \%$.

\section{RESULTADOS}

Podemos observar, na tabela 1 , o ganho de peso corporal dos animais durante as 10 semanas de estudo. Verifica-se que ocorreu aumento de peso em todos os grupos estudados. Entretanto, verifica-se que os animais treinados, quando comparados com os animais sedentários, adquiriram um menor peso corporal. Isto mostra que o modelo de natação utilizado no presente estudo favoreceu o controle de peso corporal dos animais. Na mesma tabela, foram inseridos os resultados referentes à glicemia, glicogênio do músculo gastrocnêmio e peso da gordura epididimal na condição basal. Nota-se que não houve diferença significativa na glicose sérica entre os animais. Em relação ao glicogênio muscular, verifica-se que os animais treinados regularmente, quando comparados com os animais sedentários, apresentam maior teor desse substrato no músculo. A gordura epididimal foi significativamente maior entre os ratos administrados com o esteróide e reduzida nos animais treinados.
Tabela 1. Ganho de peso corporal $(\Delta p)$, glicose sérica, glicogênio músculo gastrocnêmio e peso do tecido adiposo epididimal no final de 10 semanas de experimento nos diferentes grupos de ratos.

\begin{tabular}{lcccc}
\hline & $\begin{array}{c}\text { CS } \\
(n=10)\end{array}$ & $\begin{array}{c}\text { CT } \\
(n=10)\end{array}$ & $\begin{array}{c}\text { DxS } \\
(n=10)\end{array}$ & $\begin{array}{c}\text { DxT } \\
{[n=10)}\end{array}$ \\
\hline$\Delta p(g)$ & $149 \pm$ & & & \\
& 15 & $129 \pm 30$ & $143 \pm 26$ & $122 \pm 23$ \\
Glicose & $130 \pm$ & & & \\
sérica & 15 & $125 \pm 17$ & $121 \pm 13$ & $115 \pm 17$ \\
(mg/dL) & & & & \\
Glicogênio & & & & \\
muscular & $0.69 \pm$ & $0,86 \pm$ & $0,60 \pm$ & $0,94 \pm$ \\
(mg/100g & 0,10 & $0,10^{\mathrm{a}}$ & $0,03^{\mathrm{b}}$ & $0,18^{\mathrm{a}, \mathrm{c}}$ \\
p.c.) & & & & \\
Peso tecido & & & & \\
adiposo & $0,61 \pm$ & $0,41 \pm$ & $0,68 \pm$ & $0,41 \pm$ \\
(mg/100g & 0,18 & $0,08^{\mathrm{a}}$ & $0,20^{\mathrm{b}}$ & $0,06^{\mathrm{a}, \mathrm{c}}$ \\
p.c.) & & & & \\
\hline
\end{tabular}

Valores expressos com média \pm desvio padrão.

CS - controle sedentário; CT - controle treinado; DxS - dexametasona sedentário; $D x T$ - dexametasona treinado. $n=$ número de animais por grupo. $a . \neq C S ; b . \neq C T ; c . \neq D x S ; d . \neq D x T . p<0,05$.

$\mathrm{Na} 9^{\mathrm{a}}$ semana de estudo os animais foram submetidos ao teste de tolerância à insulina (ITT). Verificase que os ratos sedentários que receberam a dexametasona (figura 1), tiveram uma menor taxa de desaparecimento da glicose, quando comparados aos demais grupos, demonstrando resistência à insulina.

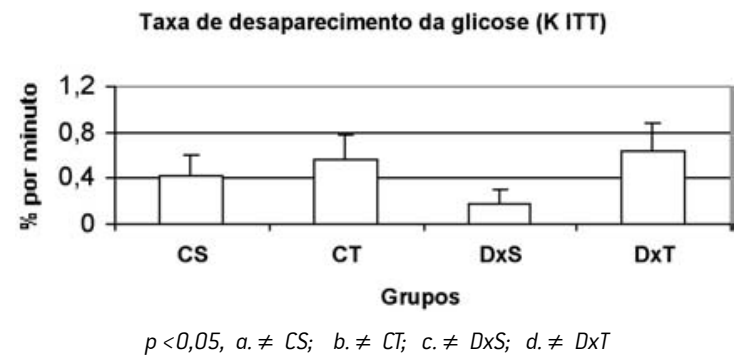

Figura 1. Taxa de desaparecimento da glicose (KITT) após 60 minutos do teste [\% por minuto] dos ratos na $9^{\circ}$ semana do experimento. Resultados expressos como média \pm desvio padrão. ( $C S=$ controle sedentário; $C T=$ controle treinado; $D \times S=$ dexametasona sedentário; $D x T$ = dexametasona treinado).

Na tabela 2 são apresentadas as concentrações plasmáticas de hormônio adrenocorticotrófico. Os níveis de $\mathrm{ACTH}$ não foram diferentes entre os grupos. Tal 
resultado indica que a aplicação crônica de baixas doses de dexametasona não interferiu no eixo hipotálomo-pituitária na condição basal. Nesta tabela foram inseridos também os resultados referentes à concentração de ácido ascórbico e do peso fresco da glândula adrenal dos animais. Observa-se, que o grupo dexametasona sedentário apresenta concentração de vitamina $C$ adrenal significativamente maior quando comparado aos outros grupos estudados. Após 10 semanas de experimento, foi verificada diferença significativa para o peso fresco da glândula adrenal somente entre o grupo dexametasona sedentário, que apresentou menor peso comparado ao grupo controle treinado.

Tabela 2. Parâmetros relacionados com o eixo hipotálamo-pituitária-adrenal avaliados no final de 10 semanas de experimento nos diferentes grupos de ratos, em condições de repouso.

\begin{tabular}{lcccc}
\hline & $\begin{array}{c}\text { CS } \\
(n=10)\end{array}$ & $\begin{array}{c}\text { CT } \\
(n=10)\end{array}$ & $\begin{array}{c}\text { DxS } \\
(n=10)\end{array}$ & $\begin{array}{c}\text { DxT } \\
(n=10)\end{array}$ \\
\hline ACTH $(\mathrm{pg} / \mathrm{mL})$ & $\begin{array}{c}90,73 \pm \\
41,42\end{array}$ & $\begin{array}{c}101,94 \pm \\
39,34\end{array}$ & $\begin{array}{c}106,27 \pm \\
38,11\end{array}$ & $\begin{array}{c}116,29 \pm \\
57,02\end{array}$ \\
\hline & & & & \\
Ácido ascórbico & $40,31 \pm$ & $34,70 \pm$ & $63,34 \pm$ & $41,30 \pm$ \\
$(\mu \mathrm{g} / \mathrm{mg})$ & 12,71 & 7,36 & $9,51^{\mathrm{a} b}$ & $6,66^{\mathrm{c}}$ \\
\hline Peso da adrenal & $4,97 \pm$ & $5,72 \pm$ & $3,79 \pm$ & $4,68 \pm$ \\
$(\mathrm{mg} / 100$ g p.c.) & 1,25 & 1,17 & $1,01^{\mathrm{b}}$ & 1,23 \\
\hline
\end{tabular}

Valores expressos com média \pm desvio padrão. CS - controle sedentário; CT controle treinado; DxS - dexametasona sedentário; DxT - dexametasona treinado, $n=$ número de animais por grupo. $a . \neq C S ; b . \neq C T ; c . \neq D x S ; d . \neq D x T . p<0,05$

Os resultados após o exercício agudo foram dispostos na tabela 3. Evidencia-se um aumento nos níveis de ACTH no grupo controle, em relação aos demais grupos. A diferença nos valores entre os animais controles e sedentários administrados com dexametasona indica possível supressão sobre o eixo hipotálamopituitária-adrenais em resposta ao uso crônico desta droga. No entanto, as concentrações semelhantes entre os grupos treinados sugerem que o exercício físico foi favorável na modulação do referido eixo. Em relação ao acido ascórbico da adrenal, semelhante aos resultados obtidos em repouso, o grupo dexametasona sedentário apresentou maior nível deste substrato ao término do exercício, confirmando a hipótese de ter ocorrido menor síntese de hormônios do estresse. O peso da adrenal foi também discretamente menor nesses animais, o que indica possível atrofia da glândula com a continuidade do tratamento com dexametasona.

Tabela 3. Parâmetros relacionados com o eixo hipotálamo-pituitária-adrenal avaliados no final de 10 semanas de experimento nos diferentes grupos de ratos, imediatamente após a sessão aguda de exercício físico.

\begin{tabular}{|c|c|c|c|c|}
\hline & $\begin{array}{c}C S \\
(n=10)\end{array}$ & $\begin{array}{c}\text { CT } \\
{[n=10)}\end{array}$ & $\begin{array}{c}\text { DxS } \\
(n=10)\end{array}$ & $\begin{array}{c}\text { DxT } \\
{[n=10)}\end{array}$ \\
\hline ACTH $(\mathrm{pg} / \mathrm{mL})$ & $\begin{array}{l}2016 \pm \\
1102,12\end{array}$ & $\begin{array}{c}286,14 \pm \\
121,34^{\mathrm{a}}\end{array}$ & $\begin{array}{c}368,51 \pm \\
113,99^{3}\end{array}$ & $\begin{array}{c}392,95 \pm \\
179,01^{3}\end{array}$ \\
\hline $\begin{array}{l}\text { Ácido ascórbico } \\
(\mu \mathrm{g} / \mathrm{mg})\end{array}$ & $\begin{array}{c}29,12 \pm \\
3,33\end{array}$ & $\begin{array}{c}26,13 \pm \\
4,05\end{array}$ & $\begin{array}{c}33,69 \pm \\
2,03^{a, b}\end{array}$ & $\begin{array}{c}27,26 \pm \\
2,94^{c}\end{array}$ \\
\hline $\begin{array}{l}\text { Peso da adrenal } \\
\text { (mg/100g de peso } \\
\text { corporal) }\end{array}$ & $\begin{array}{c}5,16 \pm \\
1,27\end{array}$ & $\begin{array}{c}5,84 \pm \\
1,10\end{array}$ & $\begin{array}{c}4,19 \pm \\
0,49\end{array}$ & $\begin{array}{c}5,35 \pm \\
0,99\end{array}$ \\
\hline
\end{tabular}

Valores expressos com média \pm desvio padrão.

CS - controle sedentário; CT - controle treinado; DxS - dexametasona sedentário; $D x T$ - dexametasona treinado, $n=$ número de animais por grupo. a. $\neq C S ; b . \neq C T ; c . \neq D x S ; d . \neq D x T . p<0,05$.

\section{DISCUSSÃO}

O uso de glicocorticóides ocorre com freqüência na população, sendo uma das drogas mais prescritas e utilizadas em todo mundo, em várias condições clínicas $(5,14)$. Consideráveis evidências têm sido acumuladas, indicando que os glicocorticóides induzem resistência à insulina e inúmeras desordens metabólicas, quando secretados ou administrados em excesso, o que ocorre em endocrinopatias e tratamentos clínicos, tanto em humanos quanto em animais. Entretanto, a prática crônica de exercícios pode resultar em benefícios a saúde, favorecendo ajustes metabólicos no sentido de promover a homeostase do organismo, mesmo após a administração de hormônios esteróides.

Dentre as análises metabólicas realizadas, o ganho ou perda excessiva de peso podem ser indicativos importantes para adequação do treinamento físico e/ou administração de glicocorticóides. No presente estudo, verificou-se que os animais treinados adquiriram peso corporal semelhante aos controles duran- 
te as 10 semanas de experimento. Tal resultado demonstra que o treinamento físico não afetou negativamente os animais e reforça os possíveis benefícios do treinamento sobre a redução da gordura corporal, uma vez que os animais treinados apresentaram menor acúmulo de tecido adiposo epididimal, quando comparados aos grupos sedentários. Este fato talvez possa justificar as observações de Francischi et al. (15), Baker \& Brownell (16) e Ciolac \& Guimarães (17), quando afirmam que o treinamento aeróbio regular colabora com a perda de gordura. Tal tentativa de analogia e de interpretação pode ser reforçada pelos resultados obtidos por Mensink et al (18), os quais evidenciaram que a intervenção de um programa de atividade física promove melhora no perfil metabólico em indivíduos diabéticos não insulino dependentes, indicando melhoria na capacidade de utilização de ácidos graxos pelo músculo esquelético.

Os glicocorticóides exercem importante ação sobre o metabolismo dos carboidratos. Acarretam aumento da glicemia, atuando na captação, consumo periférico e produção de glicose $(19,20)$. Em nosso trabalho, não detectamos diferenças significativas na glicose sérica dos animais na condição de repouso. Entretanto, o exercício físico realizado durante 10 semanas promoveu o aumento da reservas glicídicas do músculo gastrocnêmio, evidenciado pelo maior depósito de glicogênio muscular entre os animais treinados. Esta adaptação é extremamente importante para o trabalho físico, uma vez que o nível inicial deste substrato pode determinar o tempo de surgimento da fadiga, sendo que o aumento pode prorrogar a realização da atividade física, melhorando assim a performance (21).

A sensitividade insulínica, estimada pelo teste de tolerância à insulina, foi significativamente reduzida nos ratos sedentários administrados com dexametasona. Estes resultados estão de acordo com Severino et al. (1) que observaram, após 30 minutos da infusão de insulina, redução significativa da sensitividade à insulina nos ratos tratados com dexametasona, quando comparados com o grupo controle nos $6^{\circ}, 12^{\circ}$ e $26^{\circ}$ dias de experimento. Similar resultado foi obtido pelos autores, quando estimaram a sensibilidade à insulina através do "steady-state" de glicose plasmática durante a infusão glicose/insulina. A glicemia, durante as 3 horas de teste, foi significativamente maior nos ratos administrados com o esteróide. Os mecanismos pelos quais os glicocorticóides exercem seus efeitos ainda não estão completamente esclarecidos. É possível que a dexametasona atue diretamente em tecidos periféricos, resultando na resistência à insulina, ou, alternativamente, através de mudanças na concentração plasmática de glicose e de ácidos graxos livres, como observado durante a administração de glicocorticóide (22).

A resistência à insulina, em decorrência do uso de glicocorticóides, por sua vez, pode ser provocada por alterações nos receptores da insulina ou nas enzimas envolvidas nas vias de sinalização, sugerindo um mecanismo molecular multifatorial para a resistência ao hormônio no hipercortisolismo $(20,23)$.

Entretanto, a prática de atividade física pode resultar em alterações metabólicas, que podem favorecer a entrada de glicose na célula (24). O exercício físico regular tem sido importante no controle da glicemia em pessoas insulinoresistentes. Kunitomi et al. (25) reportaram que os exercícios realizados com baixa intensidade, mas por longos períodos, têm efeito favorável no controle da glicemia em indivíduos diabéticos não insulino-dependentes. Houmard et al. (26) demonstraram que, em indivíduos com sobrepeso e obesos submetidos a diferentes intensidades e volume de treinamento, a ação da insulina, mensurada durante o teste de tolerância à glicose intravenosa, foi mais eficaz nos fisicamente ativos, independente do protocolo de treinamento, quando comparados aos indivíduos sedentários.

Em nosso estudo, verifica-se que, durante o teste de tolerância à insulina, os animais treinados apresentaram tendência a menores concentrações de glicose, quando comparados ao grupo controle, indicando um possível aumento na captação dessa hexose da corrente sanguínea. Além do mais, a menor taxa de desaparecimento da glicose durante o teste, evidenciado entre os animais sedentários, e a ação efetiva do exercício físico regular em aumentar a captação de glicose pelos tecidos periféricos, confirmam as evidências da atuação positiva do treinamento físico na melhora da sensibilidade à insulina.

Além das implicações sobre o metabolismo intermediário, a administração de glicocorticóides provoca alterações sobre o funcionamento do eixo hipotálo- 
mo-hipófise, com conseqüente redução na secreção de cortisol pelas adrenais. $\mathrm{O}$ uso crônico destes esteróides sintéticos pode suprimir o ACTH, e a retirada abrupta pode encontrar o organismo deficiente em corticosteróide endógeno. Por outro lado, o exercício físico pode ser responsável pela modulação da secreção de ACTH e ser capaz de promover respostas favoráveis ao organismo submetido a situações estressantes.

Em nosso estudo, não foram observadas diferenças significativas nos níveis de ACTH plasmático entre os animais na condição de repouso. É provável que a baixa dose de dexametasona administrada não tenha exercido supressão da atividade do eixo HPA. Assim sendo, nossos resultados estão de acordo com a afirmativa de Severino et al. (1) de que a administração de baixas doses de dexametasona não provoca drásticos efeitos catabólicos e completa inibição da secreção de ACTH. Isto leva-nos a pensar que, provavelmente nessa concentração, os efeitos deletérios da dexametasona são minimizados.

Evidencia-se também, em nossos resultados, que não houve alterações nos níveis de ACTH entre os animais treinados. O treinamento sistemático pode ser responsável por uma modulação da secreção de ACTH, fazendo com que a resposta aguda ao exercício seja atenuada $(27,28)$. Jovens estudantes submetidos ao treinamento em ciclo-ergômetro durante 7 semanas apresentaram um menor aumento nas concentrações séricas de ACTH pela ação do exercício agudo quando comparado ao encontrado no período pré-treinamento (27). Segundo Viru et al. (29), esta é uma adaptação protetora ao organismo, em resposta a um feedback negativo induzido pelo cortisol e pela depleção do "pool" de ACTH da hipófise anterior. Possivelmente, em nosso estudo houve uma adaptação do eixo hipotálomo-hipófise, caracterizado pelo menor aumento do ACTH entre os grupos treinados submetidos ao esforço agudo. Esta resposta sugere que adaptações ao exercício aeróbio regular estão associadas com redução da atividade do eixo HPA. O efeito deletério dos glicocorticóides suprimindo hipotálomo-pituitária pode ser detectado também ao nível adrenal (30). A redução na secreção de ACTH pode resultar no comprometimento ou retardo do desenvolvimento desta glândula. Assim, a atrofia da adrenal seria uma maneira de identificar um possível comprometimento na secreção endógena de cortisol. Por outro lado, a estimulação crônica do córtex adrenal, pela ação do ACTH, pode gerar adaptações sobre esta glândula, fazendo com que a mesma apresente-se hipertrofiada (31). Isto pode ser detectado por aumento da massa, ou seja, do seu peso fresco (32). Diversos autores vêm utilizando o peso fresco da glândula adrenal como indicativo de estresse crônico $(33,34,35)$.

$\mathrm{O}$ modelo de treinamento físico utilizado em nosso experimento não promoveu a hipertrofia da glândula, mostrando não ser estressante ao organismo.

Entretanto, parece exercer papel importante no funcionamento do eixo HPA, prevenindo a supressão causada pelo glicocorticóide. O resultado do presente estudo confirma tal possibilidade, já que os animais treinados não apresentaram diferenças significativas nas concentrações de ACTH na condição de repouso e o mesmo aconteceu em relação ao peso da adrenal, independentemente da administração da dexametasona. No entanto, nos animais sedentários que receberam a droga, evidencia-se discreta tendência a um menor peso da adrenal no final do experimento. $\mathrm{Na}$ tentativa de encontrar evidências que pudessem demonstrar qualquer efeito negativo do uso crônico da dexametasona na funcionalidade do eixo HPA, avaliou-se os teores de ácido ascórbico da glândula adrenal dos animais. O consumo ou redução das concentrações de tal substrato pode indicar sua contribuição para a síntese de corticosterona (9). Em nosso estudo, observamos diferença significativa na concentração de ácido ascórbico na condição de repouso. Verifica-se que os animais do grupo dexametasona sedentário apresentam maior concentração deste substrato, quando comparados aos demais grupos estudados. Com este resultado, a hipótese de ter havido diminuição da secreção endógena de $\mathrm{ACTH}$, em resposta à administração da dexametasona, não deve ser desprezada. Além disso, verifica-se que não houve alterações na concentração deste substrato entre os outros animais com a prática crônica de natação, confirmando que a atividade física regular em nosso experimento não proporcionou estresse crônico.

Diante da hipótese de ter havido comprometimento funcional do eixo HPA dos animais sedentários que foram administrados com dexametasona, conforme evidenciado pelo menor consumo de ácido ascórbico 
da adrenal, decidiu-se avaliar os parâmetros descritos anteriormente após a realização de exercício físico. Tal situação pode apontar eventual comprometimento na ativação do eixo hipotálomo-pituitária-adrenais.

No presente estudo, verifica-se que os animais do grupo controle sedentário apresentam significativos aumentos da concentração de ACTH logo após a realização de 1 hora de natação, comparativamente aos ratos treinados. Tal fato indica que uma sessão única de natação, em nosso modelo, pode gerar um estímulo suficiente para alterar a síntese e secreção deste hormônio da hipófise anterior. Estes resultados estão de acordo com outras pesquisas científicas que mostraram aumentos significativos dos níveis de ACTH após a realização de esforço agudo $(8,28,36)$.

Entretanto, observa-se no presente trabalho que os níveis de ACTH do grupo sedentário que recebeu a dexametasona não se elevaram igualmente ao grupo controle após o exercício agudo, e tiveram valores semelhantes aos dos animais treinados. Tal observação indica que a administração da dexametasona interferiu negativamente sobre a atividade hipotalâmica e, conseqüentemente, hipofisiária e adrenal desses animais sedentários. Por outro lado, o exercício físico sistemático preveniu qualquer prejuízo funcional sobre o referido eixo, conforme observado pelos níveis semelhantes de ACTH após o esforço físico entre os ratos treinados. Assim, é provável que na situação de exercício agudo ocorram adaptações favoráveis que impedem a supressão do referido eixo pelo uso da dexametasona. Lac et al. (37) verificaram que os níveis de ACTH são alterados pelo exercício físico intenso, com aumento de $553 \%$ acima do nível de repouso com placebo e $350 \%$ e $146 \%$ com baixa e alta-dose de dexametasona, respectivamente. Com o intuito de investigar o mecanismo de estimulação da secreção de ACTH durante o exercício, alguns investigadores têm mensurado os níveis plasmáticos dos maiores secretagogos de ACTH: o hormônio liberador de corticotropina (CRH) e arginina vasopressina (AVP) $(38,39)$. Existem evidências de aumento dos níveis de ACTH e AVP em resposta ao exercício agudo $(7,39,40)$. Tal fato levou estudiosos a pesquisarem o comportamento do eixo hipotálomo-pituitária-adrenais em resposta à supressão da administração da dexametasona em situações de exercício.
Em estudo bastante interessante, Martignoni et al. (41) combinaram os efeitos da atividade física e a situação de hipóxia na função adrenocortical em indivíduos saudáveis. Durante a expedição científica ao Himalaia mensuraram os níveis de cortisol em condições basais e após a administração de $1 \mathrm{mg}$ de dexametasona. O estudo foi desenvolvido antes e após 15 dias de intensa caminhada na altitude. Verificou-se que nem todos os indivíduos tiveram reduzidos os níveis de cortisol após os 15 dias de caminhada. O mais interessante é que, na alta atitude, os níveis de cortisol foram significativamente altos, sugerindo que a hipóxia e o exercício físico exercem papéis relevantes sobre a secreção hormonal.

Deuster et al. (8) observaram que homens e mulheres moderadamente treinados, em resposta ao exercício a $90 \%$ do consumo máximo de oxigênio (VO2máx), não tiveram completa supressibilidade do $\mathrm{ACTH}$, quando o exercício é precedido pela administração de $4 \mathrm{mg}$ de dexametasona. Além do mais, quando a intensidade foi aumentada para $100 \%$ do VO2máx, escaparam da supressão, tanto do ACTH, como do cortisol. Estes achados indicam que o exercício físico intenso pode preponderar sobre o feedback negativo do glicocorticóide e aumentar, consideravelmente, a responsividade do eixo HPA.

Assim sendo, não se descarta a possibilidade do treinamento do presente estudo ter interferido positivamente sobre o feedback negativo da dexametasona. É possível que o exercício aeróbio também tenha modulado as secreções de CRH e AVP, favorecendo o funcionamento do eixo HPA inibido pela dexametasona. Acreditamos, no entanto, que o assunto deva ser melhor investigado.

Quanto ao peso e ao conteúdo de ácido ascórbico da adrenal, semelhante à condição de repouso, o peso fresco da adrenal foi discretamente menor no grupo dexametasona sedentário após o exercício físico.

Quanto aos níveis de ácido ascórbico, observa-se que foram, mais uma vez, maiores nos ratos dexametasona sedentários, confirmando a possibilidade de ter ocorrido menor síntese e/ou liberação de corticosterona pelas adrenais dos animais.

Portanto, nossos resultados sugerem que o exercício de natação interferiu favoravelmente na funcionalidade do eixo HPA, em resposta ao uso concomitante da dexametasona. Os níveis semelhantes de ACTH, 
peso da glândula adrenal e concentração de ácido ascórbico, entre os animais treinados, permitem nos dizer que o treinamento físico pode ser importante quando se faz uso de glicocorticóides.

Concluímos que baixas concentrações de dexametasona promovem inúmeras alterações no metabolismo e na funcionalidade do eixo HPA, que, em longo prazo, podem ser acentuadas. Porém, o treinamento físico pode amenizar os efeitos adversos do uso crônico desta droga.

\section{CORRESPONDÊNCIA}

\section{José Rodrigo Pauli}

Rua XV de Novembro, 1701, centro

13400-370 Piracicaba, S.P.

BRASIL

rodrigosere@yahoo.com.br

\section{BIBLIOGRAFIA}

1. Severino C, Brizzi P, Solinas A, Secchi G, Maioli M, Tonolo G. (2002). Low-dose dexamethasone in the rat: a model to study insulin resistance. American Journal of Physiology 283: E367-373.

2. Stojanovska L, Rosella G, Proietto J. (1990) Evolution of dexametasone-induced insulin resistance in rats. American Journal of Physiology 258: E748-756.

3. Schneiter P, Tappy L. (1998). Kinetics of dexamethasoneinduced alterations of glucose metabolism in health humans. American Journal of Physiology 275: E806-E813.

4. Kahn BB, Flier JS. (2000). Obesity and insulin resistance. The Journal of Clinical Investigation 106 (4): 473-481.

5. Hochberg Z.; Pacak K.; Chorousos G.P. (2003). Endocrine withdrawal syndromes. Endocrine Reviews 24 (4): 523-538.

6. Wittert GA, Stewart DE, Graves MP, Ellis MJ, Evans MJ, Wells JE, Donald RA, Espiner EA. (1991). Plasma corticotrophin releasing factor and vasopressin responses to exercise in normal man. Clinical Endocrinology 35: 311-317.

7. Inder WJ, Hellemans J, Swanney MP, Prickett TCR, Donald RA. (1998). Prolonged exercise increases peripheral plasma ACTH, CRH, and AVP in male athletes. Journal of Applied Physiology 85 (3): 835-841.

8. Deuster PA, Petrides JS, Singh A, Lucci EB, Chrousos GP, Gold PW. (1998). High intensity exercise promotes escape of adrenocorticotropin and cortisol from suppression by dexamethasone: sexually dimorphic responses. Journal of Clinical Endocrinology and Metabolism 83: 3332-3338.

9. Azevedo JRM (1994). Determinação de parâmetros bioquímicos em ratos sedentários e treinados após exercício agudo de natação. Tese de Doutorado. Departamento de Fisiologia e Biofísica, Universidade Estadual de Campinas, Campinas.

10. Lundbaek K (1962). Intravenous glucose tolerance as a tool in definition an diagnosis of diabetes mellitus. British Medical Journal 3: 1057-1063.

11. Henry R.J.; Cannon D.C.; Wilkeman J. (1974). Clinical Chemistry, principles and techniques. New York: Ed. Harper and Harper Row Publishes.

12. Dubois B, Gilles KA, Hamilton JK, Rebers PA. (1956) Colorimetric method for determination of sugar and related substances. Analytical Chemistry 28: 350-356.

13. Mindlin RL, Butler AM. (1938). The determination of ascorbic acid in plasma. A micromethod. Journal of Biological Chemistry 122: 673-686.

14. Bosscher K, Berghe WV, Haegeman G. (2003). The interplay between the glucocorticoid receptor and nuclear factor-kB or activator protein-1: molecular mechanisms for gene repression. Endocrine Reviews 24 (4): 488-522.

15. Francischi RP, Pereira LO, Lancha Júnior AH. (2001). Exercício, comportamento alimentar e obesidade: revisão dos efeitos sobre a composição corporal e parâmetros metabólicos. Revista Paulista de Educação Física 15 (2): 117-140.

16. Baker CW, Brownell KD. (2003). Atividade física e manutenção da perda de peso: mecanismos fisiológicos e psicológicos. In: C. Bouchard Atividade Física e Obesidade. São Paulo: Ed. Manole.

17. Ciolac EM, Guimarães GV. (2004). Exercício físico e síndrome metabólica. Revista Brasileira de Medicina do Esporte 10 (4): 319-324.

18. Mensink M, Blaak EE, Vidal H, De Bruin TWA, Glatz JFC, Saris WHM. (2003). Lifestyle changes and lipid metabo- 
lism gene expression and protein content in skeletal muscle of subjects with impaired glucose tolerance. Diabetologia 46: 1082-1089.

19. Tounian P, Schneiter P, Henry S, Delarue J, Tappy L. (1997) Effects of dexamethasone on hepatic glucose production and fructose metabolism in healthy humans. American Journal of Physiology 273 (2): E315-E320.

20. Carvalho CRO, Saad MJA. (1998). Resistência à insulina induzida por glicocorticóides: investigação de mecanismos moleculares. Arquivos Brasileiros de Endocrinologia e Metabologia 42 (1): 13-21.

21. Fairchild TJ, Armstrong AA, Rao A, Liu H, Lawrence S, Fournier PA. (2003). Glycogen synthesis in muscle fibers during active recovery from intense exercise. Medicine and Science in Sports and Exercise 35 (4): 595-602.

22. Tappy L, Randin D, Vollenweider P, Vollenweider L, Paquot N, Scherrer U, Scheneiter P, Nicod P, Jéquier E. (1994). Mechanisms of dexamethasone-induced insulin resistance in health humans. Journal of Clinical Endocrinology and Metabolism 79: 1063-1069.

23. Sakoda $H$, Ogihara T, Anai M, Funaki M, Inukai K, KatagiriI H, Fukushima Y, Onishi Y, Ono H, Fujishiro M, Kikuchi M, Oka Y, Asano T. (2000). Dexametasona-induced insulin resistance in 3T3-L1 adipocytes is due to inhibition of glucose transport rather than signal transduction. Diabetes 49: 1700-1708.

24. Luciano E, Carneiro EM, Carvalho CRO, Carvalheira JBC, Perez SB, Reis MAB, Saad MJA, Boschero AC, Velloso, LA. (2002). Endurance training improves responsiveness to insulin and modulates insulin signal transduction through the phosphatidylinositol 3-Kinase/ Akt-1 pathway. European Journal of Endocrinology 147: 149-157.

25. KunitomiI M, Takahashi K, Wada J, Suzuki H, Miyatake N, Ogawa S, Ohta S, Sugimoto H, Shikata K, Makino H. (2000). Re-evaluation of exercise prescription for Japanese type 2 diabetic patients by ventilatory threshold. Diabetes Research and Clinical Practice 50: 109-115.

26. Houmard JA, Tanner CJ, Slentz CA, Duscha BD, Mccartney JS, Kraus WE. (2004). Effect of the volume and intensity of exercise training on insulin sensitivity. Journal of Applied Physiology 96: 101-106.

27. Tabata I, Atomi Y, Mutoh Y, Miyashita M. (1990). Effect of physical training on the responses of serum adrenocorticotropic hormone during prolonged exhausting exercise. European Journal of Applied Physiology 61 (3-4): 188-192.

28. Heitkamp HCH, Schultz H, Röcker K, Dickhuth HH. (1998). Endurance training in females: changes in $\beta$ endorphin and ACTH. International Journal of Sports Medicine 19: 260-264.

29. Viru M, Litvinova L, Smirnova T, Viru A. (1994). Glucocorticoids in metabolic control during exercise: glycogen metabolism. Journal Sports Med Phys Fitness 34: (4): 377-382.

30. Lima JG, Nobrega LHC, Nobrega MLC, Rodrigues Jr AB, Pereira AFF. (2002). Supressão hipotálomo-hipófise-adrenal e risco de insuficiência adrenal secundária devido ao uso de dexametasona nasal. Arquivos Brasileiros de Endocrinologia \& Metabologia 46 (2): 193-196.

31. Junqueira LC, Carneiro J. (1995). Histologia básica. Rio de Janeiro: Ed. Guanabara Koogan.

32. Kjaer RM. (1998). Adrenal medulla and exercise training. European Journal of Applied Physiology 77:195-199.
33. Walker CD, Scribner KA, Stern JS, Dallman MF. (1992). Obese Zucker $(\mathrm{fa} / \mathrm{fa})$ rats exhibit normal target sensitivity to corticosterone and increased drive to adrenocorticotropin during diurnal trough. Endocrinology 131: 2629-2637.

34. Perhonen M, Takala T, Huttunen P, Leppaluoto J. (1995). Stress hormones after prolonged physical training in normo-and hipobaric conditions in rats. Journal of Sports Medicine 16 (2): 73-77.

35. Pauli JR, Silva ASR, Voltarelli FA, Ferreira L, Santhiago V, Romero CEM, Machado CEP, De Almeida Leme JAC, Gomes RJ, Osares AR, Luciano E. (2003). Efeitos do treinamento físico em água moderadamente fria sobre os parâmetros fisiológicos de ratos durante o exercício de natação. Revista Logos 11: 78-83.

36. Kraemer WJ, Fleck S, Callister R, Shealey M, Dudley M, Maresh CM, Cruthirds C, Murray T, Falkel JE. (1989). Training responses of plasma beta-endorphin, adrenocorticotropin, and cortisol. Medicine and Science in Sports and Exercise 21 (2): 146-153.

37. Lac G, Marquet P, Chassain AP, Galen FX. (1999). Dexamethasone in resting and exercising men. II. Effects on adrenocortical hormones. Journal of Applied Physiology 87 (1): 183-188.

38. Petrides JS, Mueller GP, Kalogeros KT, Chrousos GP, Gold PW, Deuster PA. (1994). Exercise-induced activation of the hypothalamic-pituitary-adrenal axis: marked differences in the sensitivity to glucocorticoid suppression. Journal of Clinical Endocrinology and Metabolism 79: 377-383.

39. Petrides JS, Gold PW, Mueller GP, Singh A, Stratakis C, Chrousos GP, Deuster PA. (1997). Marked differences in functioning of the hypothalamic-pituitary-adrenal axis between groups of men. Journal of Applied Physiology 82(6): 1979-1988.

40. Deuster PA, Petrides JS, Singh A, Chrousos GP, Poth M. (2000). Endocrine response to high-intensity exercise: dose-dependent effects of dexamethasone. Journal of Clinical Endocrinology and Metabolism 85 (3): 1066-1073.

41. Martignoni E, Appenzeller O, Nappi RE, Sances G, Costa A, Nappi G. (1997). The effects of physical exercise at high altitude on adrenocortical function in humans. Functional Neurology, 12 (6): 339-344. 